\title{
A New 3D Autonomous Continuous System with Two Isolated Chaotic Attractors and Its Topological Horseshoes
}

\author{
Ping Zhou ${ }^{1,2}$ and Meihua Ke ${ }^{1}$ \\ ${ }^{1}$ Key Laboratory of Network Control and Intelligent Instrument of Ministry of Education, \\ Chongqing University of Posts and Telecommunications, Chongqing 400065, China \\ ${ }^{2}$ Center of System Theory and Its Applications, Chongqing University of Posts and Telecommunications, Chongqing 400065, China
}

Correspondence should be addressed to Ping Zhou; zhouping@cqupt.edu.cn

Received 16 June 2017; Accepted 6 November 2017; Published 21 November 2017

Academic Editor: Dimitri Volchenkov

Copyright ( 2017 Ping Zhou and Meihua Ke. This is an open access article distributed under the Creative Commons Attribution License, which permits unrestricted use, distribution, and reproduction in any medium, provided the original work is properly cited.

Based on the 3D autonomous continuous Lü chaotic system, a new 3D autonomous continuous chaotic system is proposed in this paper, and there are coexisting chaotic attractors in the 3D autonomous continuous chaotic system. Moreover, there are no overlaps between the coexisting chaotic attractors; that is, there are two isolated chaotic attractors (in this paper, named "positive attractor" and "negative attractor," resp.). The "positive attractor" and "negative attractor" depend on the distance between the initial points (initial conditions) and the unstable equilibrium points. Furthermore, by means of topological horseshoes theory and numerical computation, the topological horseshoes in this 3D autonomous continuous system is found, and the topological entropy is obtained. These results indicate that the chaotic attractor emerges in the new $3 \mathrm{D}$ autonomous continuous system.

\section{Introduction}

A very interesting phenomenon in nonlinear systems is the possibility of chaos. Chaotic systems have some typical characteristics including high irregularity, unpredictability, and complexity $[1,2]$. In 1963, the first chaotic attractor in a smooth $3 \mathrm{D}$ autonomous continuous system was found by Lorenz, which is called Lorenz chaotic system [3]. As the first chaotic model, the Lorenz system reveals the complex and fundamental behaviors of the nonlinear dynamical systems. In 1999, Chen and Ueta reported another chaotic attractor in a smooth 3D autonomous continuous system named Chen chaotic system [4], which nevertheless is not topologically equivalent to Lorenz's. Afterwards, Lü and Chen carefully discussed the 3D Lorenz chaotic system and the 3D Chen chaotic system and discovered another chaotic attractor in 2002, which is called 3D Lü chaotic system [5]. The 3D Lü chaotic attractor connects the 3D Lorenz attractor and 3D Chen attractor and represents the transition from one to the other. Moreover, research on chaotic systems has attracted more and more attention in the last few decades because of its great applications in many fields like secure communication [6], data encryption [7], power system protection [8], DC motor control [8-10], flow dynamics [11], and so on [12-18].

On the other hand, coexisting chaotic attractors have been reported in many nonlinear systems in the recent years [19-23]. In [20, 21], the coexisting chaotic attractors were found in some $4 \mathrm{D}$ smooth systems, and there are overlaps between the coexisting chaotic attractors. In [22], Kengne et al. reported a simple 3D autonomous jerk system with multiple attractors; the chaotic system in [22] belongs to the generalized Lü chaotic system family. In [23], Pham et al. found the coexisting chaotic attractors in a novel $3 \mathrm{D}$ autonomous noequilibrium chaotic system, and there are overlaps between the coexisting chaotic attractors. The chaotic system in [23] belongs to the generalized Chen chaotic system family. However, there are few results on the relationship between the coexisting chaotic attractor and the initial conditions.

Motivated by the above discussions, a new 3D autonomous continuous chaotic system that has two isolated chaotic attractors (two disconnected chaotic attractors) is reported in this paper. Some basic dynamics behaviors of the new chaotic 
system such as dissipative, Lyapunov exponents spectrum, bifurcation diagram, and phase diagram are obtained. It can be found that this new chaotic system has two isolated chaotic attractors or two disconnected chaotic attractors (named "positive attractor" and "negative attractor" in this paper), which depend on the distance between the initial points (initial conditions) and the unstable equilibrium points. The necessary condition for "positive attractor" or "negative attractor" is obtained. Furthermore, the horseshoes and entropy in this $3 \mathrm{D}$ chaotic system are also discussed by means of topological horseshoes theory and numerical computation.

The outline of our paper is organized as follows. In Section 2, a new $3 \mathrm{D}$ autonomous continuous chaotic system is addressed, and some basic dynamics behaviors of the new chaotic system are yielded. In Section 3, the horseshoes and entropy for the 3D chaotic system are investigated. In Section 4 , the conclusion is given.

\section{A New 3D Autonomous Continuous Chaotic System}

Normally, the 3D autonomous continuous chaotic systems can be described by $d x / d t=A x+f(x)$. Here, $x=\left(x_{1}, x_{2}\right.$, $\left.x_{3}\right)^{T}$ is the state vector, $f(x)=\left(f_{1}(x), f_{2}(x), f_{3}(x)\right)^{T}$ is the nonlinear term of the $3 \mathrm{D}$ autonomous continuous chaotic system, and the constant matrix $A=\left[a_{i j}\right](i, j=1,2,3)$ is determined by the linear part of the $3 \mathrm{D}$ autonomous chaotic system. According to the results [24] reported by Vanecek and Celikovsk, the 3D autonomous continuous Lorenz chaotic system satisfies $a_{12} a_{21}>0$, the 3D autonomous continuous Chen chaotic system satisfies $a_{12} a_{21}<0$, and the 3D autonomous continuous Lü chaotic system satisfies $a_{12} a_{21}=$ $0\left(a_{12} \neq 0, a_{21}=0\right)$. One can obtain that the chaotic system in [22] satisfies $a_{12} a_{21}=0\left(a_{12} \neq 0, a_{21}=0\right)$, and the chaotic system in [23] satisfies $a_{12} a_{21}<0$. In this section, a new $3 \mathrm{D}$ autonomous continuous chaotic system that satisfies $a_{12}=a_{21}=0\left(a_{12} a_{21}=0\right)$ will be discussed.

The 3D Lü chaotic system [5] is described as follows:

$$
\begin{aligned}
& \dot{x}_{1}=-a_{11} x_{1}+a_{12} x_{2}, \\
& \dot{x}_{2}=a_{21} x_{1}+a_{22} x_{2}-x_{1} x_{3}, \\
& \dot{x}_{3}=x_{1} x_{2}-3 x_{3},
\end{aligned}
$$

where $a_{11}=a_{12}=36, a_{21}=0, a_{22}=20$, and $a_{12} a_{21}=0$.

Now, based on the 3D Lü chaotic system (1), a new 3D autonomous continuous system that satisfies $a_{12}=a_{21}=0$ is presented, and it is shown as follows:

$$
\begin{aligned}
& \dot{x}_{1}=-x_{1}+0.5 x_{1} x_{3}+x_{2} x_{3}, \\
& \dot{x}_{2}=a x_{2}-1.2 x_{1} x_{3}, \\
& \dot{x}_{3}=x_{1} x_{2}-6 x_{3},
\end{aligned}
$$

where $0 \leq a \leq 4$. The second and third equations in system (2) are similar to the second and third equations in system (1), respectively. But, the first equation in system (2) is different from the first equation in system (1). Obviously, $a_{12}=a_{21}=0$ in system (2). So, system (2) is different from the 3D Lü chaotic system (1).

It is easily yielded that $\partial \dot{x}_{1} / \partial x_{1}+\partial \dot{x}_{2} / \partial x_{2}+\partial \dot{x}_{3} / \partial x_{3}=$ $a-7<0$. So, the new system (2) is a dissipative system, and it can experience or develop attractors.

First, the Lyapunov exponents spectrum of system (2) with respect to parameter $a$ can be obtained by numerical calculation, which is displayed in Figure 1.

According to Figure 1, the maximum Lyapunov exponent $\lambda_{1}$ is positive for $0.06 \leq a \leq 0.84,0.91 \leq a \leq 1.35,1.45 \leq a \leq$ $1.85,2.26 \leq a \leq 3.28$, and $3.42 \leq a \leq 4$. Therefore, the chaotic attractor emerges in system (2) for suitable system parameter a.

Now, setting the parameter $a=2.5$, the five unstable equilibrium points in system (2) are $s_{0}=(0,0,0), p^{+}=$ $(3.5355,1.7202,1.0136), p^{-}=(-3.5355,-1.7202,1.0136)$, $q^{+}=(3.5355,-3.4880,-2.0553)$, and $q^{-}=(-3.5355,3.4880$, $-2.0553)$, respectively. The Lyapunov exponents are $\lambda_{1}=$ $0.5758, \lambda_{2}=0$, and $\lambda_{3}=-5.1023$, respectively. The Lyapunov dimension is $D_{L}=2+\lambda_{1} /\left|\lambda_{3}\right|=2.1129$; this result means that system (2) is fractal. Therefore, the chaotic attractor emerges in system (2) for parameter $a=2.5$. By numerical calculation, it can be found that there are two isolated chaotic attractors, which depend on the initial conditions. After a large number of numerical calculations, one has that the chaotic attractor depends on the distance between the initial points and the unstable equilibrium points $\left(p^{+}, p^{-}, q^{+}\right.$, and $\left.q^{-}\right)$. Some results are shown as follows:

(1) When the initial point $x_{0}=\left(x_{1}(0), x_{2}(0), x_{3}(0)\right)$ is closed to the unstable equilibrium point $p^{+}$or $q^{+}$, the new system (2) has the same chaotic attractor. This attractor is named "positive attractor" in this paper, which refers to $x_{1}(t)>0$. A necessary condition for a "positive attractor" is $x_{1}(0)>0$.

(2) When the initial point $x_{0}=\left(x_{1}(0), x_{2}(0), x_{3}(0)\right)$ is closed to the unstable equilibrium point $p^{-}$or $q^{-}$, the new system (2) has the same attractor. This attractor is named "negative attractor" in this paper, which refers to $x_{1}(t)<0$. A necessary condition for a "negative attractor" is $x_{1}(0)<0$.

Next, choose some initial conditions, for example.

Case 1 (the initial point $x_{0}$ is closed to unstable equilibrium point $\left.p^{+}\right)$. Let the initial conditions be $(2,2,2)$. The distance between this initial point and unstable equilibrium points $p^{+}, p^{-}, q^{+}$, and $q^{-}$is $1.8464,6.7420,6.9944$, and 7.0215 , respectively. Therefore, the initial point is closed to unstable equilibrium point $p^{+}$. So, the new system (2) has the "positive attractor," which is shown in Figure 2.

Case 2 (the initial point $x_{0}$ is closed to unstable equilibrium point $\left.p^{-}\right)$. Let the initial conditions be $(-2,-2,2)$. The distance between this initial point and unstable equilibrium points $p^{+}, p^{-}, q^{+}$, and $q^{-}$is $6.7420,1.8464,7.0215$, and 6.9944, respectively. Therefore, the initial point is closed to unstable equilibrium point $p^{-}$. So, the new system (2) has the "negative attractor," which is displayed in Figure 3.

Case 3 (the initial point $x_{0}$ is closed to unstable equilibrium point $\left.q^{+}\right)$. Let the initial conditions be $(3,-3,-2)$. The 

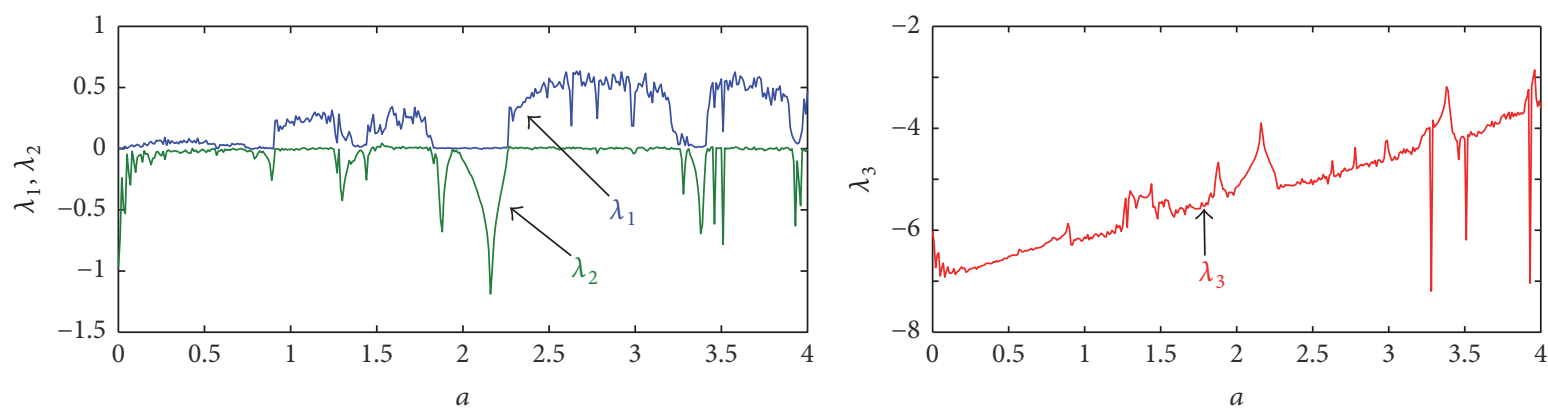

FIGURE 1: Diagram of the Lyapunov exponent spectrum varies with respect to parameter $a$.
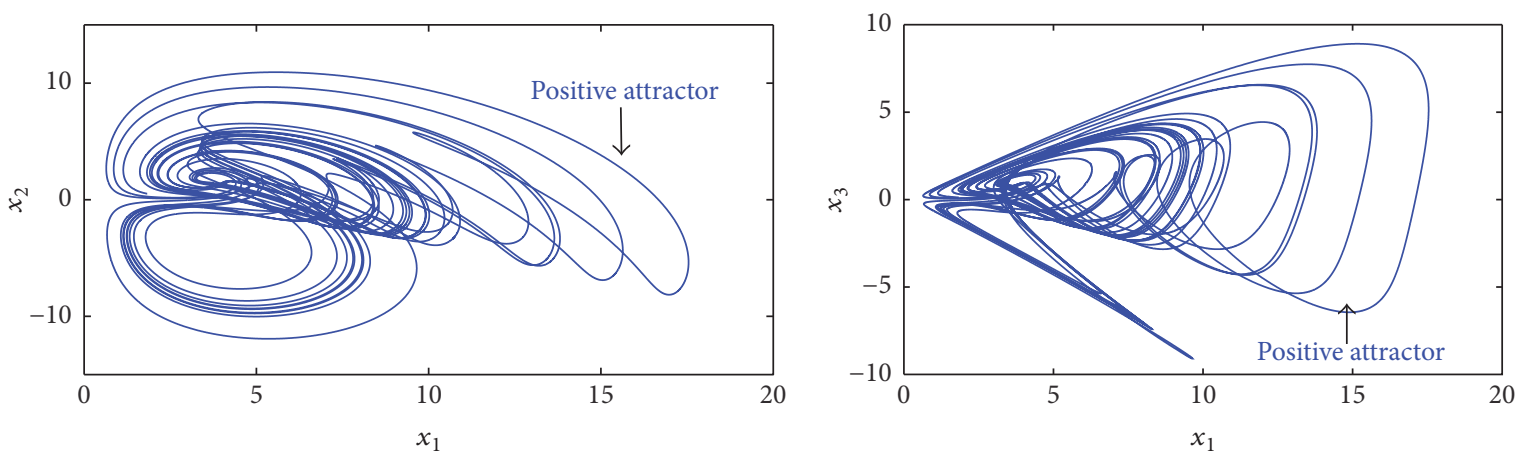

Figure 2: The "positive attractor" in system (2).
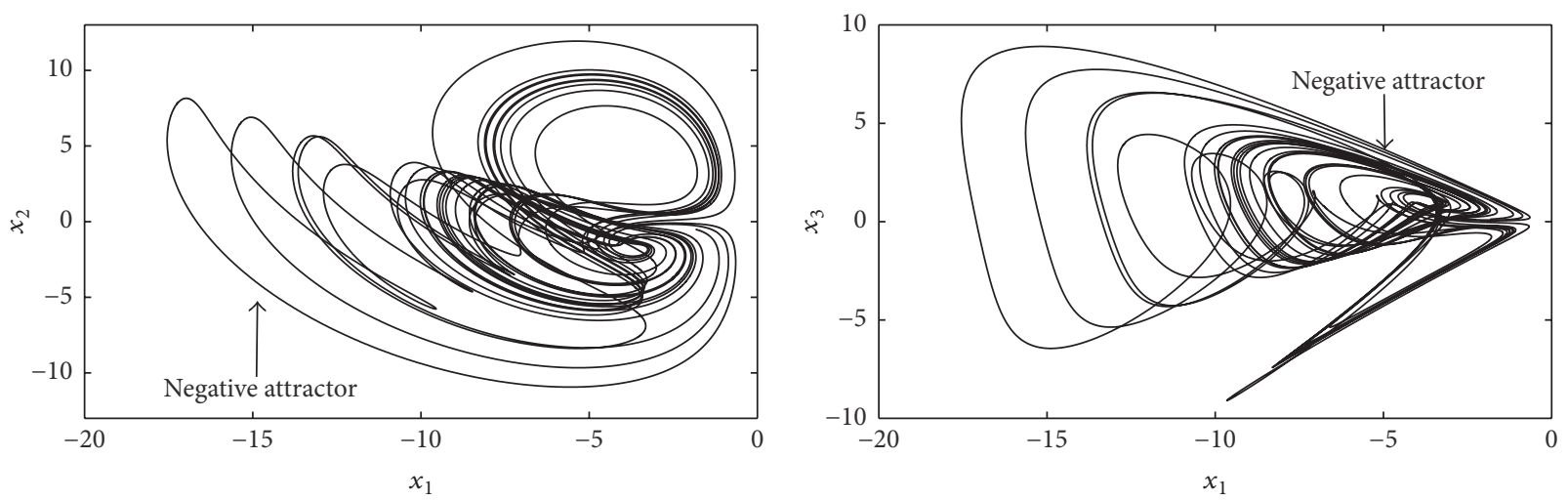

FIgURE 3: The "negative attractor" in system (2).

distance between this initial point and unstable equilibrium points $p^{+}, p^{-}, q^{+}$, and $q^{-}$is $5.6257,7.3097,0.7266$, and 9.2092, respectively. Therefore, the initial point is closed to unstable equilibrium point $q^{+}$. So, the new system (2) has the "positive attractor," which is shown in Figure 4.

Case 4 (the initial point $x_{0}$ is closed to unstable equilibrium point $\left.q^{-}\right)$. Let the initial conditions be $(-3,3,-2)$. The distance between this initial point and unstable equilibrium points $p^{+}, p^{-}, q^{+}$, and $q^{-}$is $7.3097,5.6257,9.2092$, and 0.7266 , respectively. Therefore, the initial point is closed to unstable equilibrium point $q^{-}$. So, the new system (2) has the "negative attractor," which is displayed in Figure 5.
According to Figures 2, 3, 4, and 5, we can obtain that the new system (2) has two isolated chaotic regions: one is named "positive attractor" and other one is named "negative attractor" in this paper. It is worth mentioning that there are also two isolated chaotic attractors for any other parameter $a$. For example, let $a=3.5$; the Lyapunov exponents of system (2) are $\lambda_{1}=0.5698, \lambda_{2}=0$, and $\lambda_{3}=-4.2189$, respectively. Two isolated chaotic attractors in the new system (2) are shown as Figure 6, where the initial conditions of "positive attractor" and "negative attractor" are $(3,-3,-2)$ and $(-3,3$, $-2)$, respectively.

In addition, let $a=2.2$; the Lyapunov exponents of system (2) are $\lambda_{1}=0, \lambda_{2}=-0.5250$, and $\lambda_{3}=-4.5310$, respectively. 

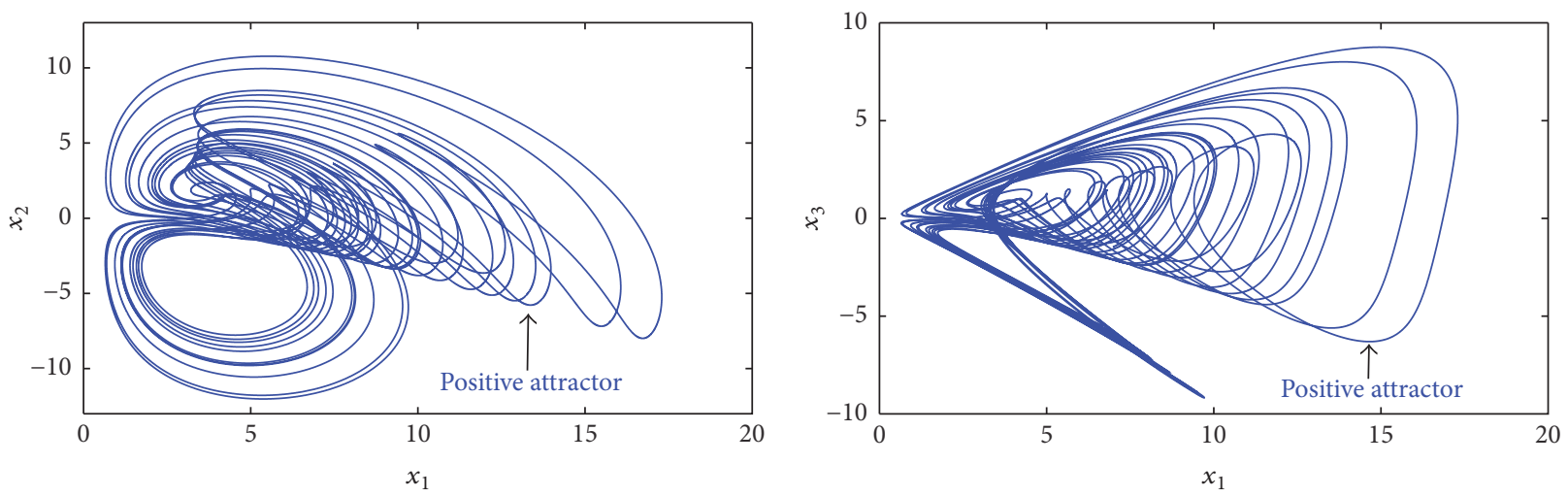

Figure 4: The "positive attractor" in system (2).
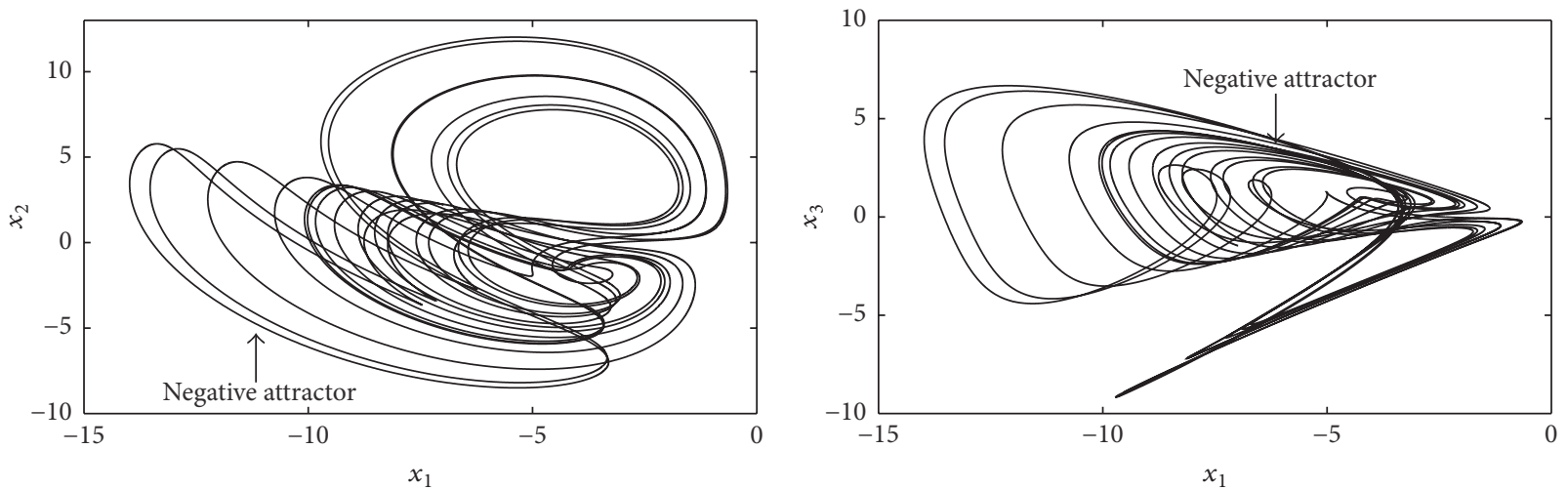

Figure 5: The "negative attractor" in system (2).
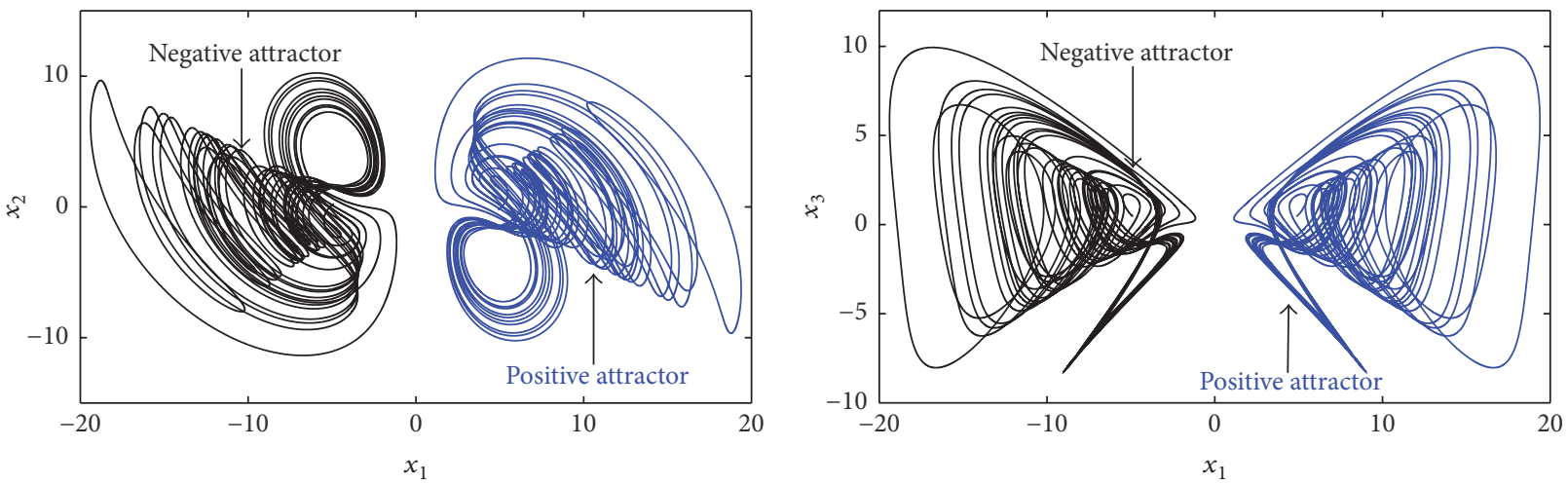

FIgURE 6: The "positive attractor" and "negative attractor" in system (2).

So, there is no chaotic attractor, and there is periodic orbit which is shown in Figure 7.

Finally, the bifurcation diagram of variables $x_{2}$ and $x_{3}$ with respect to parameter $a$ is displayed in Figure 8. It can be observed that the bifurcation diagram coincides well with the Lyapunov exponents spectrum.

Remark 1. Obviously, the Lü chaotic system (1) and system (2) in this paper are invariant under the transformation $\left(x_{1}, x_{2}, x_{3}\right) \rightarrow\left(-x_{1},-x_{2}, x_{3}\right)$. However, the geometries of chaotic attractors of the Lü chaotic system (1) and system (2) are quite different. Firstly, for a given initial condition, the state variable $x_{1}$ in the Lü chaotic system (1) can be greater than zero or less than zero. Conversely, the state variable $x_{1}$ in system (2) can only be greater than zero or can only be less than zero. Secondly, for arbitrary initial conditions, the state variable $x_{3}$ in the Lü chaotic system (1) can only be greater than zero. Conversely, the state variable $x_{3}$ in system (2) can be greater than zero or can be less than zero. 

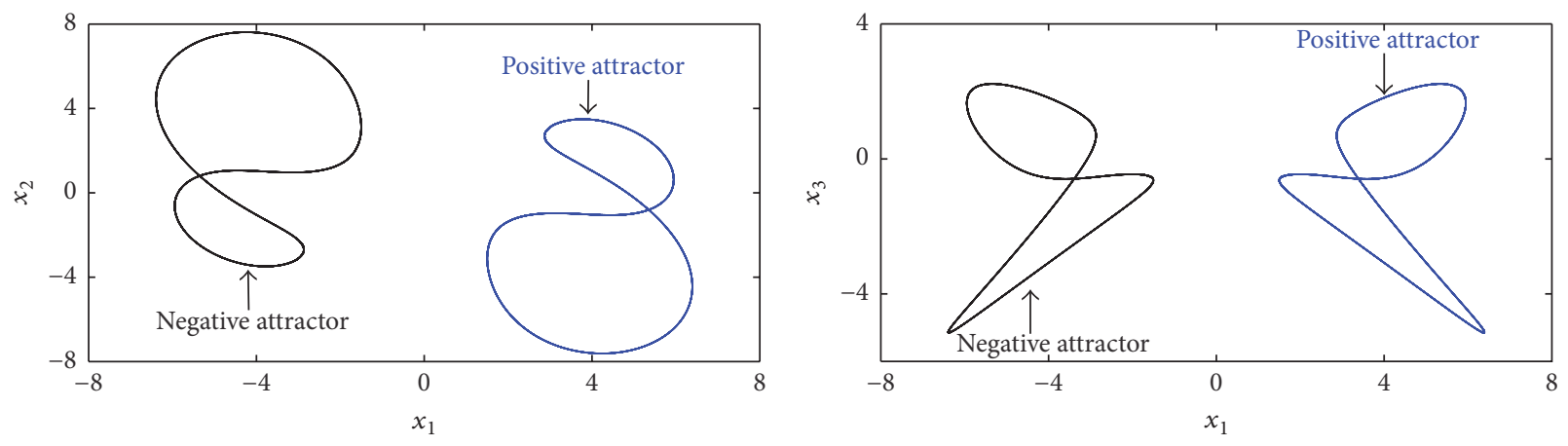

FIgURE 7: The periodic orbit in system (2) for $a=2.2$.
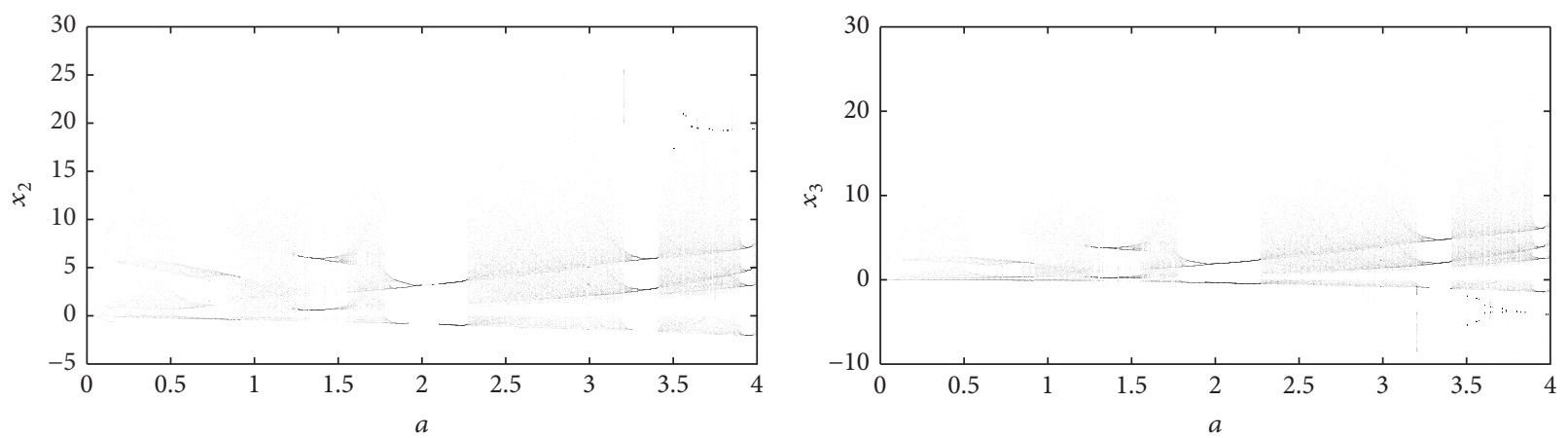

FIGURE 8: The bifurcation diagram of variables $x_{2}$ and $x_{3}$ with respect to parameter $a$.

\section{Horseshoes and Entropy in New Chaotic System (2)}

First, some theoretical criteria of topological horseshoes are recalled.

Let $X$ be a metric space, $D$ is a compact subset of $X$, and $f: D \rightarrow X$ is a map satisfying the assumption that there exist $m$ mutually disjoint compact subsets $D_{1}, D_{2}, \ldots, D_{m}$ of $D$; the restriction of $f$ to each $D_{i}$, that is, $f \mid D_{i}$, is continuous.

Definition 2 (see [25]). For each $1 \leq i \leq m$, let $D_{i}^{1}$ and $D_{i}^{2}$ be two fixed disjoint compact subsets of $D_{i}$. A connected subset $l$ of $D_{i}$ is said to connect $D_{i}^{1}$ and $D_{i}^{2}$, if $l \cap D_{i}^{1}=\varnothing$ and $l \cap D_{i}^{2}=$ $\varnothing$, and we denote this by $D_{i}^{1} \leftrightarrow D_{i}^{2}$.

Definition 3 (see [25]). Let $l \subset D_{i}$ be a connected subset; we say that $f(l)$ is suitably across $D_{j}$ with respect to $D_{j}^{1}$ and $D_{j}^{2}$, if $l$ contains a connected subset $l^{\prime}$ such that $f\left(l^{\prime}\right) \subset D_{j}, f\left(l^{\prime}\right) \cap$ $D_{j}^{1}=\varnothing$, and $f\left(l^{\prime}\right) \cap D_{j}^{2}=\varnothing$; that is, $D_{j}^{1} \stackrel{f\left(l^{\prime}\right)}{\longleftrightarrow} D_{j}^{2}$. In this case, we denote it by $f(l) \mapsto D_{j}$. In case that $f(l) \mapsto D_{j}$ holds true for every connected subset $l \subset D_{j}$ with $D_{i}^{1} \stackrel{l}{\leftrightarrow} D_{i}^{2}$, we say that $f\left(D_{i}\right)$ is suitably across $D_{j}$ with respect to two pairs $\left(D_{i}^{1}, D_{i}^{2}\right)$ and $\left(D_{j}^{1}, D_{j}^{2}\right)$, or $f\left(D_{i}\right) \mapsto D_{j}$ in case of no confusion.

Theorem 4 (see [26]). Suppose that the map $f: D \rightarrow X$ satisfies the following assumptions:
(1) There exist $m$ mutually disjoint compact subsets $D_{1}, D_{2}$, $\ldots, D_{m}$ of $D ; f \mid D_{i}$ is continuous.

(2) The relation $f\left(D_{i}\right) \rightarrow D_{j}$ holds for every pair with $i, j$ taken from $1 \leq i, j \leq m$. Then there exists a compact invariant set $K \subset D$, such that $f \mid K$ is semiconjugate to the full $m$ shift dynamics $\sigma \mid \sum_{m}$, and the topological entropy is ent $(f) \geq$ $\log m$.

Remark 5. The $m$-shift is also called the Bernoulli $m$-shift. The symbolic series space $\sum_{m}$ is the collection of all biinfinite sequences:

$$
s=\left\{\ldots, s_{-n}, \ldots, s_{-1}, s_{0}, s_{1}, \ldots, s_{n}, \ldots\right\},
$$

where $s_{i} \in\{0,1, \ldots, m-1\}$. The shift map $\sigma$ is defined as

$$
\sigma(s)=\left\{\ldots, s_{-n+1}, \ldots, s_{0}, s_{1}, s_{2}, \ldots s_{n+1}, \ldots\right\} .
$$

It is well known that $\sum_{m}$ is a Cantor set, which is compact, totally disconnected, and perfect. As a dynamical system defined on $\sum_{m}, \sigma$ has a countable infinity of periodic orbits consisting of orbits of all periods, an uncountable infinity of periodic orbits, and a dense orbit. A direct consequence of these three properties is that the dynamics generated by the shift map are sensitive to initial conditions. Mathematically, the topological entropy ent $(f)>0$ measures its complexity, which roughly means the exponential growth rate of the number of distinguishable orbits as time advances. When $m>1, \operatorname{ent}(f)>0$; therefore the system is chaotic. For 


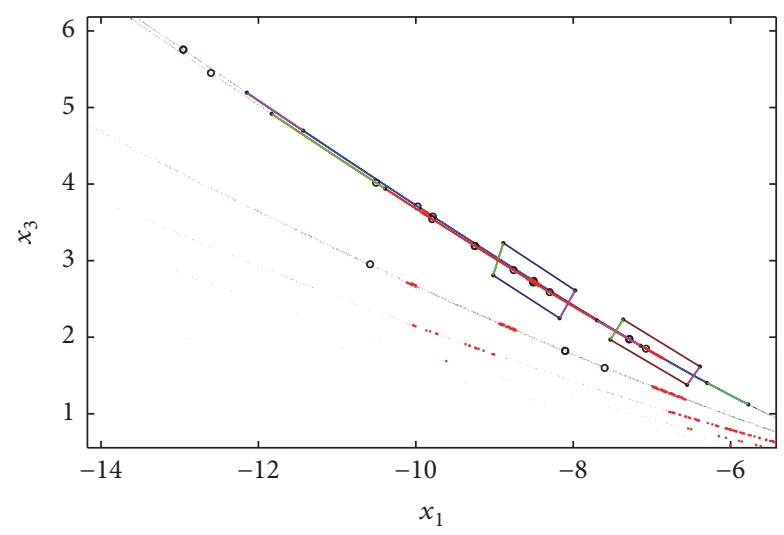

FIgURE 9: A screenshot of the MATLAB GUI program.

more details of the above symbolic dynamics and horseshoes theory, we refer the reader to [25-28].

Corollary 6 (see [27]). If $f^{p}\left(D_{1}\right) \mapsto D_{1}, f^{p}\left(D_{1}\right) \mapsto D_{2}$, $f^{q}\left(D_{2}\right) \mapsto D_{1}$, and $f^{q}\left(D_{2}\right) \mapsto D_{2}$, then there exists a compact invariant set $K \subset D$, such that $f^{p+q} \mid K$ is semiconjugate to 2 -shift dynamics, and ent $(f) \geq(1 / p+q) \log 2$.

In order to find a horseshoes in system (2) with parameter $a=2.5$ and initial conditions $(-2,-2,2)$, we will first utilize the technique of cross section and the corresponding Poincaré map. By taking the set

$$
\Pi=\left\{\left(x_{1}, x_{2}, x_{3}\right) \mid x_{2}=1.8, \dot{x}_{2}>0\right\}
$$

as a Poincaré section plane, we chose the corresponding Poincaré map $H: \Pi \rightarrow \Pi$ as follows: for each $\mathrm{x}=\left(x_{1}, x_{3}\right) \triangleq$ $\left(x_{1}, 1.8, x_{3}\right) \in \Pi, H(\mathrm{x})$ is taken to be the first return point in $\Pi$ under the flow with the initial condition $\mathrm{x}$. Then, we use a MATLAB GUI program called "A toolbox for finding horseshoes in 2D map" [27]. After many attempts, we find a topological horseshoes by a similar method proposed in [27], as shown in Figure 9.

As shown in Figure 10, we find two subsets $D_{1}$ and $D_{2}$, where the coordinates of four vertices of $D_{1}$ are

$$
\begin{aligned}
& {[-8.842643136,3.160443654],} \\
& {[-9.179288567,2.956713578],} \\
& {[-8.309621205,2.374627645],} \\
& {[-8.057137132,2.578357722] ;}
\end{aligned}
$$

and the coordinates of four vertices of $D_{2}$ are

$$
\begin{aligned}
& {[-7.362805931,2.156345421],} \\
& {[-7.503074861,1.938063196],} \\
& {[-6.703541963,1.486946599],} \\
& {[-6.605353713,1.661572378] .}
\end{aligned}
$$

Numerical computation shows that the two subsets under $H$ are continuous, and their images are illustrated in Figures $10(\mathrm{a})$ and $10(\mathrm{~b})$, respectively.

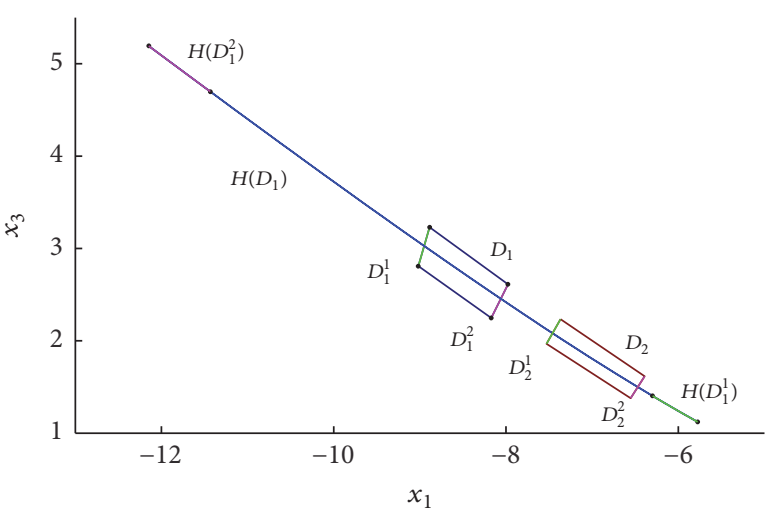

(a)

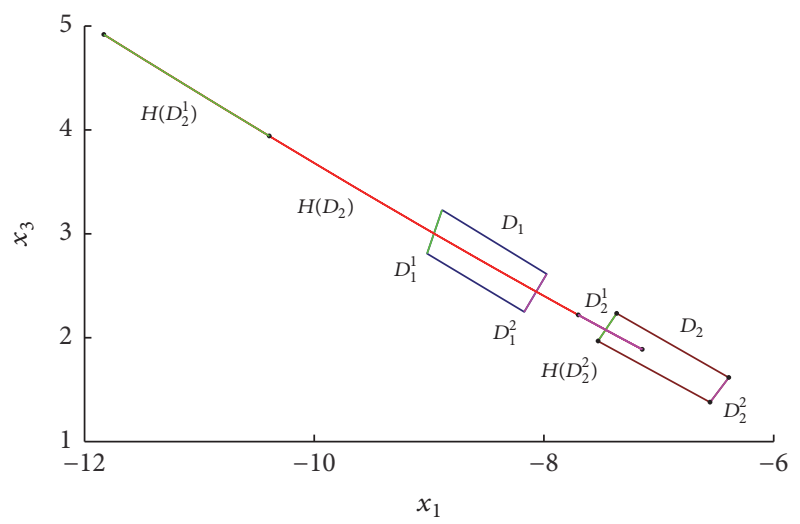

(b)

Figure 10: A new horseshoes of the map.

It is easy to see from Figure 10(a) that $H\left(D_{1}\right)$ passes through $D_{1}$ and $D_{2}$ between their top and bottom sides and transversely intersects $D_{1}$ with $D_{1}^{1}$ and $D_{1}^{2}$ and intersects $D_{2}$ with $D_{2}^{1}$ and $D_{2}^{2}$. So each connected subset $D_{1}$, if it is connection of $D_{1}^{1}$ and $D_{1}^{2}$, then its images under $H$ must be across $D_{1}$ with respect to $D_{1}^{1}$ and $D_{1}^{2}$ and across $D_{2}$ with respect to $D_{2}^{1}$ and $D_{2}^{2}$. Then we have $H\left(D_{1}\right) \mapsto D_{1}$ and $H\left(D_{1}\right) \mapsto D_{2}$. Similarly, we have $H\left(D_{2}\right) \mapsto D_{1}$ from Figure 10(b) too.

According to the topological horseshoes Corollary 6, there exists a compact invariant set $\Lambda \subset D$, such that $H^{2}$ । $\Lambda$ is semiconjugate to 2-shift dynamics and the topological entropy of $H$ is $\operatorname{ent}(H) \geq(1 / 2) \log 2$, which indicates that the map is chaotic indeed.

By the same way, a horseshoes in system (2) with parameter $a=2.5$ and initial conditions $(2,2,2)$ can be obtained. Therefore, the chaotic attractor emerges in system (2) for parameter $a=2.5$.

\section{Conclusions}

In this paper, a 3D chaotic system satisfying $a_{12}=a_{21}=0$ is suggested. Some basic dynamics behaviors such as dissipative, Lyapunov exponents spectrum, bifurcation diagram, and phase diagram are obtained. The coexisting chaotic attractors are found in this 3D chaotic system, and there are two isolated chaotic attractors (named "positive attractor" and "negative 
attractor," resp.) that depend on the distance between the initial points and the unstable equilibrium points. There are no overlaps between the "positive attractor" and "negative attractor."

Furthermore, by means of topological horseshoes theory and numerical computation, a horseshoes in system (2) with parameter $a=2.5$ is obtained. Meanwhile, we obtained that the topological entropy is ent $(f) \geq(1 / 2) \log 2$. These results indicate that the chaotic attractor emerges in system (2) for parameter $a=2.5$.

\section{Conflicts of Interest}

The authors declare that there are no conflicts of interest regarding the publication of this paper.

\section{References}

[1] G. R. Chen and X. Dong, From Chaos to order Perspectives, Methodologies and Applications, World Scientific, Singapore, 1998.

[2] J. C. Sprott, Chaos and Time-Series Analysis, Oxford University Press, New York, NY, USA, 2003.

[3] E. N. Lorenz, "Deterministic nonperiodic flow," Journal of the Atmospheric Sciences, vol. 20, no. 2, pp. 130-141, 1963.

[4] G. Chen and T. Ueta, "Yet another chaotic attractor," International Journal of Bifurcation and Chaos, vol. 9, no. 7, pp. 14651466, 1999.

[5] J. Lü and G. Chen, "A new chaotic attractor coined," International Journal of Bifurcation and Chaos, vol. 12, no. 3, pp. 659661, 2002.

[6] A. N. Miliou, I. P. Antoniades, S. G. Stavrinides, and A. N. Anagnostopoulos, "Secure communication by chaotic synchronization: robustness under noisy conditions," Nonlinear Analysis: Real World Applications, vol. 8, no. 3, pp. 1003-1012, 2007.

[7] P. Muthukumar, P. Balasubramaniam, and K. Ratnavelu, "Synchronization of a novel fractional order stretch-twist-fold (STF) flow chaotic system and its application to a new authenticated encryption scheme (AES)," Nonlinear Dynamics, vol. 77, p. 1547, 2014.

[8] J. Ni, C. Liu, K. Liu, and X. Pang, "Variable speed synergetic control for chaotic oscillation in power system," Nonlinear Dynamics, vol. 78, no. 1, pp. 681-690, 2014.

[9] D. Q. Wei, L. Wan, X. S. Luo, S. Y. Zeng, and B. Zhang, "Global exponential stabilization for chaotic brushless DC motors with a single input," Nonlinear Dynamics, vol. 77, no. 1-2, pp. 209-212, 2014.

[10] P. Zhou, R. J. Bai, and J. M. Zheng, "Stabilization of a fractionalorder chaotic brushless DC motor via a single input," Nonlinear Dynamics, vol. 82, no. 1-2, pp. 519-525, 2015.

[11] J. M. Ottino, F. J. Muzzio, M. Tjahjadi, J. G. Franjione, S. C. Jana, and H. A. Kusch, "Chaos, symmetry, and self-similarity: exploiting order and disorder in mixing processes," Science, vol. 257, no. 5071, pp. 754-760, 1992.

[12] T. Yang and L. O. Chua, "Impulsive stabilization for control and synchronization of chaotic systems: theory and application to secure communication," IEEE Transactions on Circuits and Systems I: Fundamental Theory and Applications, vol. 44, no. 10, pp. 976-988, 1997.

[13] E. Montbrió, J. Kurths, and B. Blasius, "Synchronization of two interacting populations of oscillators," Physical Review E:
Statistical, Nonlinear, and Soft Matter Physics, vol. 70, Article ID 056125, 2004.

[14] Y. Feng, Z. Wei, U. E. Kocamaz, A. Akgül, and I. Moroz, "Synchronization and electronic circuit application of hidden hyperchaos in a four-dimensional self-exciting homopolar disc dynamo without equilibria," Complexity, vol. 2017, Article ID 7101927, 11 pages, 2017.

[15] J. Ma, F. Wu, G. Ren, and J. Tang, "A class of initials-dependent dynamical systems," Applied Mathematics and Computation, vol. 298, pp. 65-76, 2017.

[16] C. Wang, R. Chu, and J. Ma, "Controlling a chaotic resonator by means of dynamic track control," Complexity, vol. 21, no. 1, pp. 370-378, 2015.

[17] M. Lv, C. Wang, G. Ren, J. Ma, and X. Song, "Model of electrical activity in a neuron under magnetic flow effect," Nonlinear Dynamics, vol. 85, no. 3, pp. 1479-1490, 2016.

[18] M. Lv and J. Ma, "Multiple modes of electrical activities in a new neuron model under electromagnetic radiation," Neurocomputing, vol. 205, pp. 375-381, 2016.

[19] J. Argyris and I. Andreadis, "On the influence of noise on the coexistence of chaotic attractors," Chaos, Solitons \& Fractals, vol. 11, no. 6, pp. 941-946, 2000.

[20] F. Yuan, G. Wang, Y. Shen, and X. Wang, "Coexisting attractors in a memcapacitor-based chaotic oscillator," Nonlinear Dynamics, vol. 86, no. 1, pp. 37-50, 2016.

[21] Q. Lai and S. Chen, "Coexisting attractors generated from a new 4D smooth chaotic system," International Journal of Control, Automation, and Systems, vol. 14, no. 4, pp. 1124-1131, 2016.

[22] J. Kengne, Z. T. Njitacke, and H. B. Fotsin, "Dynamical analysis of a simple autonomous jerk system with multiple attractors," Nonlinear Dynamics, vol. 83, p. 751, 2016.

[23] V.-T. Pham, C. Volos, S. Jafari, and T. Kapitaniak, "Coexistence of hidden chaotic attractors in a novel no-equilibrium system," Nonlinear Dynamics, vol. 87, no. 3, pp. 2001-2010, 2017.

[24] A. Vanecek and S. Celikovsk, Control Systems: From Linear Analysis to Synthesis of Chaos, Pretice-Hall, London, UK, 1996.

[25] X.-S. Yang, "Topological horseshoes and computer assisted verification of chaotic dynamics," International Journal of Bifurcation and Chaos, vol. 19, no. 4, pp. 1127-1145, 2009.

[26] X.-S. Yang, H. Li, and Y. Huang, "A planar topological horseshoe theory with applications to computer verifications of chaos," Journal of Physics A: Mathematical and General, vol. 38, no. 19, pp. 4175-4185, 2005.

[27] Q. Li and X.-S. Yang, "A simple method for finding topological horseshoes," International Journal of Bifurcation and Chaos, vol. 20, no. 2, pp. 467-478, 2010.

[28] X.-S. Yang and Y. Tang, "Horseshoes in piecewise continuous maps," Chaos, Solitons \& Fractals, vol. 19, no. 4, pp. 841-845, 2004. 


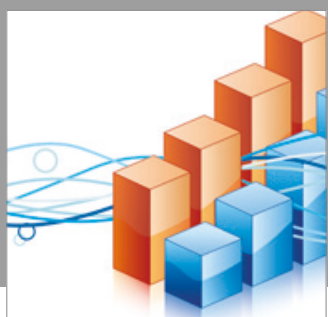

Advances in

Operations Research

vatersals

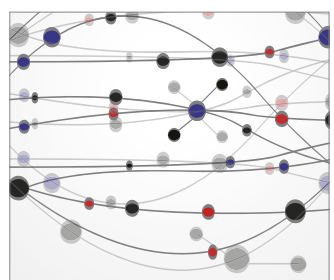

\section{The Scientific} World Journal
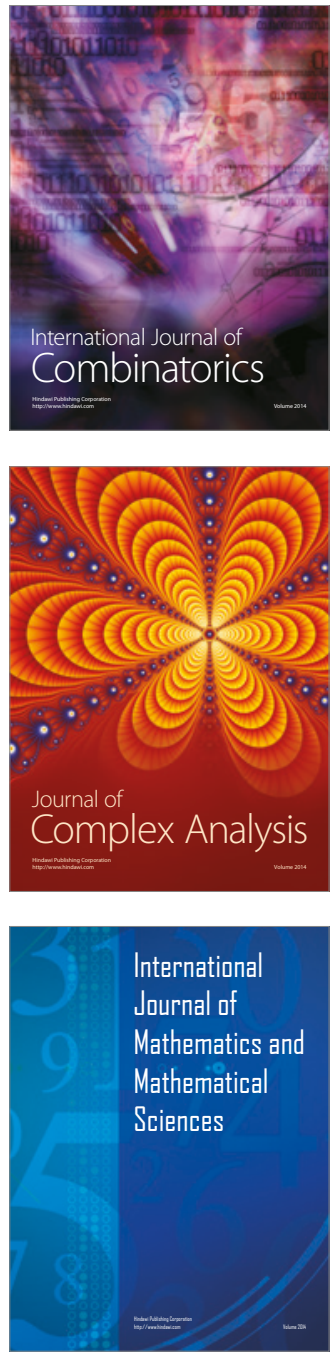
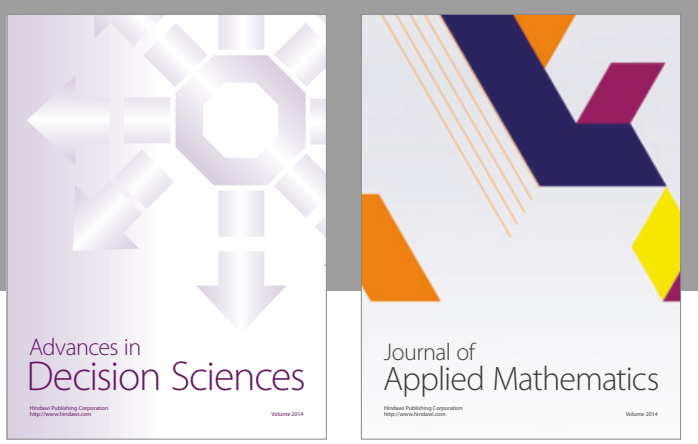

Algebra

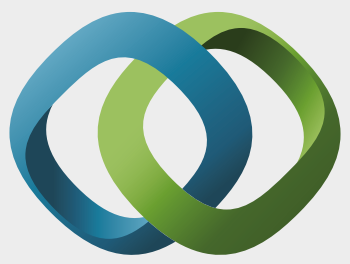

\section{Hindawi}

Submit your manuscripts at

https://www.hindawi.com
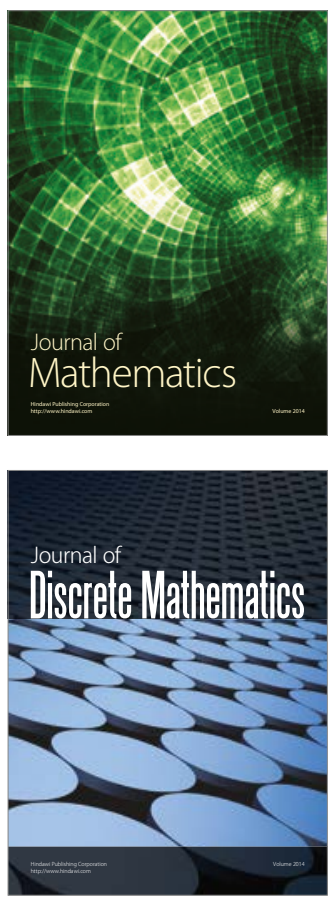

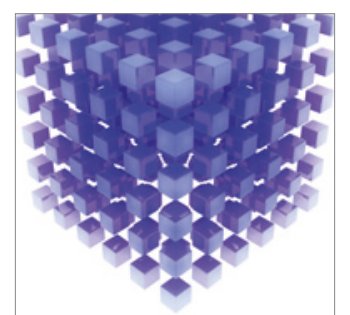

Mathematical Problems in Engineering
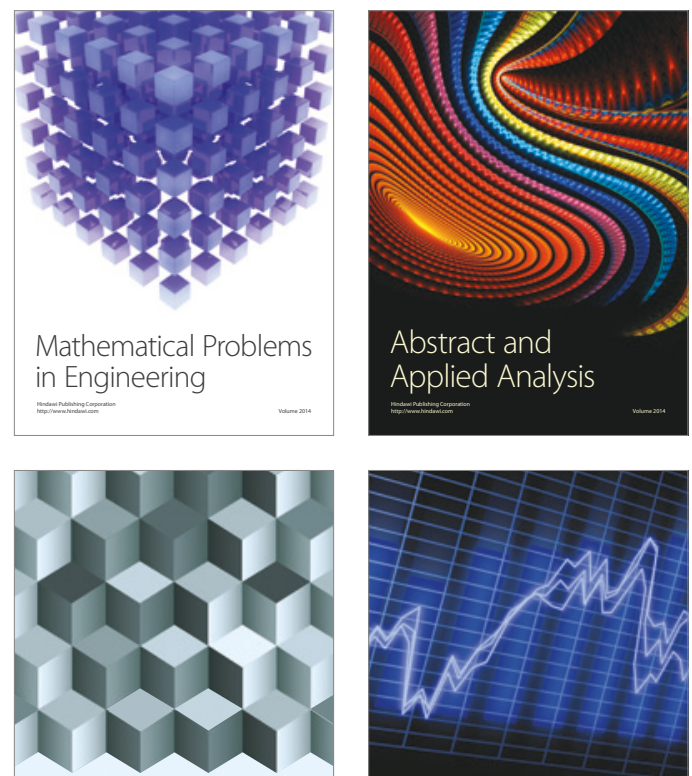

Journal of

Function Spaces

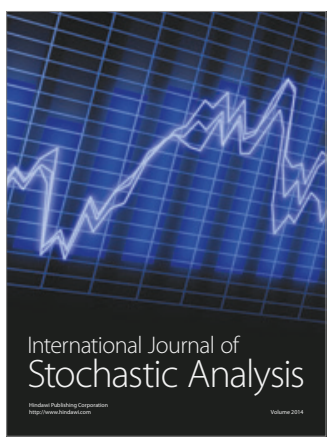

Probability and Statistics
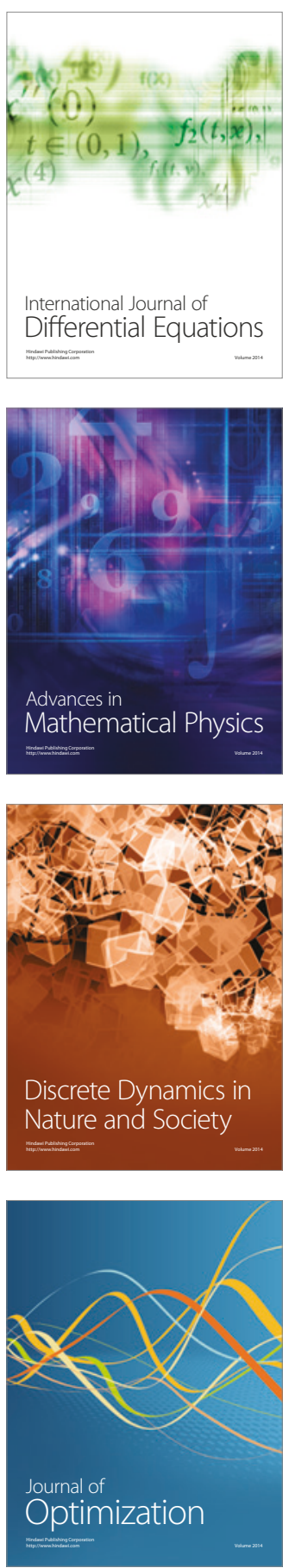